\title{
Reflexiones sobre la competencia comunicativa en los pregrados de las áreas de salud de universidades colombianas
}

\author{
Olga F. Salazar-Blanco, Cruzana Echeverri-Restrepo, Diana P. Díaz-Hernández
}

Introducción. La enseñanza de la comunicación en los programas de salud en las universidades colombianas requiere un diagnóstico porque hasta el momento no se conocen trabajos sobre esta competencia. Como un punto de partida, en el 'VII Encuentro de Educación Superior en Salud: la comunicación, un reto en la formación de los profesionales', se realizó una mesa redonda con decanos de los programas de salud de siete universidades colombianas. El objetivo era conocer el enfoque que se da a la comunicación en la formación en estos programas. El encuentro con los decanos se llevó a cabo en la ciudad de Medellín en septiembre de 2013.

Sujetos y métodos. El encuentro focal con siete decanos de instituciones con programas del área de la salud se centró en la reflexión y debate académico sobre las preguntas '¿cómo está estructurada la enseñanza de comunicación en los programas de salud de sus instituciones?' y '¿qué propuestas plantean para mejorarla?'. Las intervenciones de los participantes se registraron digitalmente, se transcribieron y se procedió a su análisis e interpretación, con el apoyo de bibliografía sobre el tema.

Resultados. Los decanos destacaron la importancia de la comunicación en la formación de los profesionales, que se desarrolla de diferentes formas en el currículo, pero sin una intencionalidad especialmente en los semestres clínicos. Destacaron el valor de la interdisciplinariedad, la contribución de las áreas sociales y de las actividades extracurriculares en la formación, y se reconoció la escasa sensibilización de los docentes por el tema.

Conclusiones. La formación en comunicación para los estudiantes de programas de salud debe ser transcurricular, intencional y explícita en el currículo, con prácticas reflexivas integradas en las discusiones clínicas.

Palabras clave. Competencia comunicativa. Currículo. Educación médica. Grado.

Reflections on communicative competence in the undergraduate health areas of Colombian universities

Introduction. Teaching of communication in health programs in some Colombian universities requires a current diagnosis. A meeting (in the frame of the 'VII Encuentro de Educación Superior en Salud: la comunicación, un reto en la formación de los profesionales') of seven deans of health programs from Colombian universities was carried with the objective to know the communication approach in undergraduate students from these institutions. This meeting was held in Medellín in September 2013.

Subjects and methods. The focal group with seven attendees responded these questions: 'How is structured in the respective health programs the communication teaching' and 'What proposals are there in your institutions to improve it?'. Answers were digital recorded and then a transcription was performed.

Results. The deans admitted the importance of communication in the teaching/learning process in their health programs. Likewise they all expressed their programs include a variety of related communication courses. Even though, they have the perception of least real impact in the behavior of their graduates. It was underlined the importance of interdisciplinary and contributions of social areas, as well as extracurricular experiences. The scarce of qualified training on the communication by teachers.

Conclusions. Communication training in health program students should be transversal during the total enrollment period of students. Even more, it should be more explicit during clinic semesters, including integrated reflexive practices, along with patients.

Key words. Clinical competence. Communication skills. Medical education. Undergraduate.
Departamento de Educación Médica (O.F. Salazar-Blanco, C. EcheverriRestrepo). Vicedecanato (D.P. DíazHernández). Grupo de Investigación Edusalud. Facultad de Medicina. Universidad de Antioquia. Medellín, Antioquia, Colombia.

Correspondencia:

Profa. Olga Francisca Salazar Blanco. Departamento de Educación Médica. Facultad de Medicina. Universidad de Antioquia. Carrera 51D, n.‥ 6229. Medellín (Colombia).

E-mail:

olga.salazar@udea.edu.co

Agradecimientos:

E. Gaviria Rivera (Universidad de Antioquia), L.J. Giraldo Múnera (Fundación Universitaria San Martín), A.M. Matijasevic (Universidad de Caldas), C.I. Vargas Castellanos (Universidad Industrial de Santander), M. Uribe Ríos (Universidad Pontificia Bolivariana), J.C. Monsalve Botero (Universidad Tecnológica de Pereira),

A. Maya Elejalde (Universidad Remington), R. Ruiz Moral (Universidad de Córdoba), F.J. Mercado (Universidad de Guadalajara) y L. Casabuenas Duarte (miembro del comité organizador del evento).

Conflicto de intereses: No declarado.

Competing interests: None declared.

(c) 2015 FEM 


\section{Introducción}

El presente artículo expresa una visión sobre la enseñanza de la comunicación en los programas de salud de universidades colombianas, construida a partir de la mesa redonda de decanos de siete facultades de medicina y programas de salud del país, en el marco del 'VII Encuentro de Educación Superior en Salud: la comunicación, un reto en la formación de los profesionales', organizado por el Grupo de Investigación Edusalud y el Departamento de Educación Médica de la Universidad de Antioquia, el mes de septiembre de 2013, en Medellín, Colombia.

El objetivo fue identificar cómo se desarrolla la competencia comunicativa en los programas de medicina de las universidades colombianas. Las preguntas orientadoras para propiciar el diálogo fueron: '¿cómo se trabaja la competencia comunicativa en los programas de salud de sus instituciones?' y '¿qué propuestas plantean para mejorarla?.'

\section{Sujetos y métodos}

Dos meses antes del encuentro de educación superior en salud, se envió una carta de invitación a los decanos de 19 facultades, de universidades públicas y privadas acreditadas y con trayectoria académica, para que participaran en este evento. Invitación que fue ampliada a todos los decanos de facultades de la ciudad sede del evento. Previo permiso de los participantes, se realizó registro con grabadora digital; posteriormente, se transcribió y se analizó. Los decanos participantes se constituyeron como el grupo focal, a partir del cual se desarrolló la investigación con enfoque cualitativo.

\section{Resultados}

Participaron decanos de cuatro universidades públicas: la Universidad de Caldas (UC), la Universidad Tecnológica de Pereira (UTP), la Facultad de Salud de la Universidad Industrial de Santander (UIS) -que representa cinco programas de pregrado en salud, posgrados en el área clinicoquirúrgica, posgrados en áreas básicas y en epidemiología en salud pública - y la Universidad de Antioquia (UdeA) -con tres programas de pregrado (medicina, instrumentación quirúrgica y atención prehospitalaria), 43 posgrados medicoquirúrgicos y una maestría en ciencias clínicas-. Las tres universidades privadas fueron: Universidad Pontificia Bolivariana de Medellín (UPB), que tiene programa de pregra- do de medicina y de enfermería, además de 23 posgrados medicoquirúrgicos; la Fundación Universitaria San Martín, seccional Medellín (FUSM), y la Universidad Remington de Medellín (UR), con programa de medicina.

$\mathrm{Al}$ encuentro asistieron integrantes del grupo de investigación, del comité organizador del evento y profesores invitados de universidades internacionales, la Universidad de Córdoba, España (UCE) y la Universidad de Guadalajara (UG), que dieron sus aportes en base a la experiencia en sus países. A continuación se presentan las categorías producto del análisis del encuentro.

Un primer aspecto, reconocido por todos los participantes, fue la presencia e importancia de la comunicación en la formación de los profesionales de la salud y su inclusión en los currículos, aunque reconocen que ha sido escaso el impacto logrado en la formación de sus profesionales:

'Nosotros tenemos la ventaja de haber creado un programa nuevo y desde un principio pensamos en cuáles eran las necesidades de formación en nuestro médico y definimos en ese momento como siete necesidades de formación, y dos de ellas eran: considerar que el médico es un educador y el médico es un comunicador basados en las expectativas del paciente.' (FUSM)

'Tenemos una serie de contenidos con los cuales tratamos de fortalecer y desarrollar ese aspecto de la comunicación de nuestro estudiante con el paciente, con el individuo enfermo o con el individuo sano o con la comunidad en general y lo llevamos a una práctica' (UR)

Estas aseveraciones están en consonancia con la formación en comunicación en otros países en los cuales han incluido la competencia comunicativa y se tienen los consensos sobre ella [1-6]

Algunas de las razones que sustentan la percepción de que no se materialice la comunicación efectiva como competencia fundamental del profesional de la salud son: la insuficiente reflexión de los docentes y administrativos sobre el tema; la estructura que tienen los currículos, que dan más importancia a otros elementos del contexto propios de los modelos dominantes de salud; la escasa sensibilización de los docentes por el tema; la concepción de la medicina, predominantemente bajo un modelo biomédico, y el rol social del médico, que sigue siendo paternalista ante un amplio grupo de profesionales, aspectos que también refieren otros autores, como Borrell y Tizón $[7,8]$. 
'Como docentes nunca hemos reflexionado sobre la comunicación dentro de la enseñanza del acto médico. Puntualmente, no existe una asignatura; sin embargo, si es un tema que sale a flote muchas veces en las reflexiones de consejo de facultad. Curiosamente, y sobre todo cuando llegaron los problemas, cuando hubo quejas por la agresión entre docentes y estudiantes, o entre estudiantes, o cuando hubo una conducta irrespetuosa con un paciente, entonces fue cuando comenzó a plantearse esa problemática.' (UIS)

Otro aspecto en el cual se presentaron acuerdos, con diferentes matices, es sobre cómo debe estar presente la competencia comunicativa en el currículo; se concluyó que debe ser transversal en el proceso formativo y, en este sentido, debería estar integrada y ser visible durante toda la carrera y no sólo en cursos específicos.

'Seguimos convencidos de que esto no es un curso; la idea de la formación en comunicación no es alrededor de un curso, sino que tiene que ser un eje transversal que esté inmerso en todas las actividades que hacemos.' (FUSM)

En este sentido, existe concordancia con la concepción que se abriga en la educación médica actual sobre el desarrollo de esta competencia y la importancia que tienen, para la adquisición de la competencia comunicativa y la formación integral, las actividades extracurriculares, los cursos flexibles y de humanidades, los eventos culturales y otros espacios fuera del aula, así como las experiencias previas y las vivencias de cada estudiante en su proceso formativo, que determinan en su conjunto el aprendizaje de los conocimientos, habilidades y actitudes que conforman la competencia [9-11].

'Transversalmente, a lo largo de la carrera, se está trabajando con una familia y haciendo un diagnóstico del estado de salud en esa comunidad. Entonces aparecen otras cosas que tienen que ver con el manejo o la capacidad crítica para el manejo, el análisis y la aplicación de la información, y le vamos dando otra serie de elementos en los diferentes semestres.' (FUSM)

Sin embargo, reconocen que algunos de estos espacios extracurriculares y flexibles para el aprendizaje de la comunicación no son valorados como procesos trascendentes en la formación profesional por los estudiantes y docentes, los consideran de poca relevancia para su desempeño.
'Nuestros estudiantes tienen opcionales; algunas de éstas son en educación, comunicación, desarrollos de las nuevas tecnologías, etc., pero ellos lo ven como algo de relleno, algo que no es parte de su formación integral.' (UIS)

De acuerdo con el perfil profesional que se busca, deben orientarse las actividades y estrategias que integren la comunicación durante todo el programa, articularse en los cursos básicos y clínicos y en las actividades fuera del aula. Se resalta la importancia de adaptar el programa, los propósitos de formación al tipo de médico de cada institución: un médico más comunitario o familiar, un médico general o social, o un médico investigador. Como dice Palés, el diseño de los programas debe partir de los resultados que se esperan en el profesional [12].

'Nuestro médico tiene que ser educador y un comunicador porque pensamos que la comunicación es la base de la relación médico-paciente, y ésta no se limita simplemente a hacer un diagnóstico, sino que va algo más allá dentro de esa interacción que se debe dar entre el médico y el paciente.' (FUSM)

Otro punto para desarrollar la competencia comunicativa, rescatado por varios participantes, es contar con universidades que tienen programas de humanidades, los cuales ofrecen cursos para los estudiantes de las áreas de la salud, ya que se considera de gran importancia la interdisciplinariedad y los aportes de los profesionales de las áreas sociales como la antropología, la sociología, la comunicación y la educación en el proceso formativo de los estudiantes.

'La gran ventaja que tenemos es la interdisciplinariedad y lo que todas las ciencias sociales aporten a la formación de los otros pregrados.' (UPB)

Otra forma, referida por los participantes, para trabajar la interdisciplinariedad es la realización de trabajos a lo largo de la carrera, con diferentes actividades que se van haciendo complejas en la medida en que los estudiantes avanzan en el proceso educativo, desde ser observadores de las actuaciones de otros, en diferentes escenarios, hasta ser ellos verdaderos participantes en los encuentros clínicos y aplicar los aprendizajes previos de habilidades clínicas y de tecnologías de la información y la comunicación, hacer el registro de la información de forma manual o electrónica e integrar conocimientos sobre promoción de la salud y educación, con los pacientes, familias y comunidades. 
Se observó la intencionalidad de la enseñanza de la comunicación durante los primeros semestres, con la presencia de cursos o actividades enfocadas básicamente a mejorar la comunicación escrita y oral; sin embargo, no se evidencia una clara intencionalidad hacia el desarrollo posterior de la competencia específica de comunicación en salud: la adquisición de las habilidades de comunicación interpersonal, que incluya la relación con el paciente, con otros profesionales de la salud y con la comunidad en general, habilidades que posiblemente se perfeccionen durante los semestres de práctica clínica, en los que los estudiantes tienen contacto continuo con los diferentes actores del proceso salud-enfermedad. Falta trabajar la comunicación en sus lógicas de sentido, que trae retos a la comunicación asertiva, entre colegas y expertos en dialogo disciplinar e interdisciplinar, y con las comunidades en diálogo de saberes, llegando a los pacientes y familiares con un lenguaje asequible para la comprensión de la promoción de la salud, la prevención de la enfermedad, el tratamiento y la rehabilitación. Llegar al otro en su lenguaje, con explicaciones al alcance de su lógica y emociones, saber explicar, saber mediar para hacerse entender.

Aunque se reconoce la necesidad de que la comunicación sea una competencia transversal, a la hora de desarrollarla en el currículo sólo se hace explícita con algunos cursos de comunicación, que hacen énfasis en los medios de comunicación, como son las técnicas del lenguaje escrito, los medios alternativos de comunicación organizacional (carteles, comunicados, informes técnicos), sin que logren constituir una comunicación asertiva en el acto médico; además, se cuestionan si el momento y la forma de darlos es la más adecuada.

'En el primer semestre trabajamos habilidades comunicativas con los estudiantes. Tenemos un contenido donde se trabaja todo lo que tiene relación con las habilidades de lectoescritura porque pensamos que nuestros estudiantes están llegando hoy día con serias deficiencias en lectoescritura y comunicación.' (FUSM)

A partir del encuentro, emergieron algunas fortalezas y debilidades sobre el desarrollo de la competencia comunicativa en la formación de los profesionales de la salud. Entre las fortalezas se encontró que en todos los programas se dispone de cursos y actividades enfocadas al aprendizaje de algunas habilidades comunicativas; sin embargo, falta mayor intencionalidad e integración en las actividades clínicas porque no se evidencian objetivos explícitos de aprendizaje en los últimos semestres, en los que incluso -afirman los decanos- se puede llegar al desaprendizaje de conceptos y actitudes aprendidas en los cursos de humanidades, de comunicación y otros trabajados durante los primeros semestres.

Otra de las fortalezas es que algunas instituciones cuentan con prácticas de pocos estudiantes por tutor, que favorecen el proceso de aprendizaje por medio de la relación que se establece con los estudiantes, la realimentación y el seguimiento del proceso de aprendizaje a lo largo del semestre.

También se destaca la existencia de centros de simulación en consultorios para entrevistas con pacientes estandarizados y otras propuestas activas de aprendizaje.

'Tenemos un semillero de estudiantes que se han preparado como pacientes estandarizados; como ya conocen las patologías y vieron medicina interna, están preparados y son nuestro recurso para arrancar, posiblemente este semestre, con un proyecto de investigación para hacer el proceso pedagógico.' (UC)

Con respecto a las debilidades encontradas, una de ellas se relaciona con los inconvenientes originados por la actual ley en salud de Colombia, la Ley 100, que llevó a la reducción de los escenarios de práctica y, en algunos casos, a la exclusión de las universidades para hacer las prácticas en instituciones que atienden a usuarios de empresas promotoras de salud privadas.

La técnica expositiva del acto médico descontextualiza la relación médico-paciente y evapora la comunicación en cuanto no hay tiempo de discutir dinámicas de la comprensión, la interpretación, el reconocimiento del otro en sus entornos culturales, hábitos, actos, costumbres, no permite al médico darse a entender y además lo limita en su conocimiento del otro, que es persona, enfermo, y no enfermedad, un genérico. Al paciente lo incomunica en sus emociones, reduciendo su ser al objeto de consulta, la parte, el fragmento en disfunción de la salud.

Conjuntamente, se ve como una limitación el hecho de que incluso hoy se tiene una concepción de la comunicación como algo innato al ser humano, heredado, que depende de cada uno; no se ve como una competencia para aprender; aspecto que también refieren otros autores, como una dificultad en la formación en comunicación [13].

Del mismo modo, se analizaron las dificultades propias de la brecha generacional entre docentes y estudiantes, que hace alusión a la tecnología en el encuentro clínico, como una dificultad: la presencia 
del ordenador para el registro electrónico de la historia clínica, que interfiere en el contacto visual con el paciente; también, las ayudas diagnósticas que reemplazan la palabra, el tacto en el encuentro, que se basa en dar diagnósticos y a veces no se tiene en cuenta el deseo, el sentir del paciente. Posiblemente, la dificultad esté no en la presencia del ordenador, una herramienta que puede facilitar el registro de la información, sino en el uso que se le dé a ésta, y preservar la buena comunicación del profesional de la salud con su paciente.

Una de las debilidades que es común a muchas de las universidades es la ausencia de una intencionalidad explícita en la formación de esta competencia; no está como algo pensado para desarrollar de forma transcurricular, donde se reconozcan los momentos en los cuales se está trabajando y evaluando la comunicación. Consideran los decanos que si bien, probablemente, se está trabajando en este aspecto y se reconoce que aparecen algunas actividades y propósitos de formación -porque, como plantea la Escuela de Palo Alto, es imposible no comunicar, la no comunicación no existe, como no existe la no relación $[9,14]-$, no se reflexiona sobre su rol dentro del proceso educativo.

En la evaluación de la comunicación se perciben algunas debilidades relacionadas con las estrategias, el tipo e instrumentos de evaluación que se hace durante y al final del proceso educativo. Aunque algunas instituciones refieren esfuerzos por asignarle un valor o un porcentaje a la comunicación en cursos específicos o durante toda la carrera, existen dificultades en el uso de los instrumentos por parte de los docentes porque probablemente no tienen la capacitación o la convicción para hacerlo. En algunos casos se evalúan aspectos de la comunicación que no se han visibilizado como logros del estudiante. La evaluación formativa debería estar presente en todo el proceso educativo y contar con herramientas e instrumentos objetivos [15-19].

'Nosotros sí lo evaluamos en las prácticas. Hay un porcentaje de la evaluación que es un $10 \%$. Se evalúa cómo se comunica el estudiante con el paciente y cómo comunica al paciente y a las familias. Cuando está efectuando la ronda médica, una de las cosas que tiene que hacer es comunicar y tiene que estar dentro del proceso de comunicación, pero prima la subjetividad de la evaluación del docente.' (UPB)

Algunos señalaron la intencionalidad en la evaluación de los aprendizajes del área, que incluye habilidades y actitudes, pero también anotaron la falta de capacitación en los docentes para hacer una evaluación más objetiva.

Otra dificultad es la subvaloración que hacen los estudiantes a los aspectos que se evalúan durante el proceso; sólo rescatan el valor que tiene la comunicación en la formación de profesionales de la salud al final, o cuando ya han egresado del programa, probablemente porque tampoco los docentes reconocen la trascendencia de las discusiones y reflexiones que se realizan en las prácticas, sobre lo que representa evaluar el proceso de interacción de los estudiantes con los pacientes; por ejemplo, cuando dejan solo al estudiante haciendo una historia clínica y luego evalúan la historia que presenta, pero no la comunicación con el paciente.

En la comunicación hay una mediación significativa que repercute en el reconocimiento de roles entre el paciente y el médico, así como en el posicionamiento profesional, la calidad en la atención, la identidad en la dignidad del otro, que es interpretado, leído, comprendido.

En algunas instituciones se cuenta con laboratorios de simulación y programas para el aprendizaje de la comunicación mediante el uso de entrevistas simuladas y con pacientes estandarizados, pero no se tiene claro cómo se va a evaluar el aprendizaje de estas habilidades. Además, desde la percepción de los decanos, no es garantía de un buen uso y un buen resultado sin el convencimiento de los docentes sobre el alcance real de la comunicación en la formación de los estudiantes; el avance es más lento, pero es un indicador para trabajar en ello a través de eventos de capacitación y actualización.

Entonces, la evaluación de la competencia comunicativa constituye una carencia en la formación tanto de los estudiantes como de los docentes.

'Nos quejamos de los muchachos de hoy. Decimos de ellos que son la generación del pulgar y nosotros, enseñados a la conferencia magistral, que es la que nos da créditos, que es la que hemos hecho toda la vida, ¿por qué no utilizamos lo que ellos saben en el escenario de clase? Trabajando con las tecnologías de la información y la comunicación, enseñándoles a consultar. Lo único que hacemos es regañarlos: «póngame atención», «apague el móvil», "¿por qué está chateando ahí?», «¿a quién está llamando?».' (FUSM)

Algunos participantes se centraron en las debilidades de los estudiantes y las particularidades de ellos, en el mundo de la comunicación con la tecnología del móvil, las bases de datos e Internet accesibles a estos equipos, sumados a las deficiencias que traen y a las carencias de la formación básica. Parecería 
que nuevamente la brecha generacional no permite una adecuada utilización de las tecnologías de la información y la comunicación como una de las fortalezas generacionales, las cuales podrían usarse en beneficio de la comunicación con los pacientes, entre profesionales y en los procesos de aprendizaje $[20,21]$ y llegar más a los estudiantes, con estrategias que ellos utilizan, por lo que es necesario que los docentes se actualicen en estos aspectos para favorecer la comunicación entre los estudiantes y los pacientes e impactar positivamente en su salud. Esto es parte de la propuesta de la formación en comunicación, que trascienda el proceso educativo en la universidad; una formación centrada en quien aprende es más fácil que luego se traduzca en una atención centrada en quien consulta y parte del reconocimiento como persona, del trabajo por el desarrollo de sus capacidades, del autorreconocimiento de su emoción, de su saber y del acompañamiento para desarrollarlo como sujeto y profesional.

Esta visión de la importancia del docente en los aprendizajes de y con los estudiantes, de la bidireccionalidad del aprendizaje, debería considerarse parte de la concepción del ser docente, de ser un buen comunicador y un apasionado por el aprendizaje, dispuesto a aprender y desaprender contenidos, tecnologías y actitudes [22-27].

'Necesitamos un tipo de profesional que también tiene que aprender habilidades de tecnología de la información, aprovechar que las generaciones de ahora son realmente habilidosas en esto y que, además, son una gran manera de enganchar y de motivar al alumno, lo cual evidentemente lleva a tener una capacitación por parte de los profesores en este sentido.' (UCE)

Aunque se considera como una debilidad la falta de formación de los profesores en comunicación, también se reconocen esfuerzos de las diferentes instituciones por darles oportunidades, herramientas pedagógicas y didácticas en el área, aunque otros refieren que la comunicación no está presente en los programas de capacitación docente y ello ocasiona dificultades en la relación de docentes y estudiantes.

'Por política, en la universidad, todos los docentes debemos hacer un diplomado en docencia universitaria, de 120 horas, que cuenta con tres contenidos temáticos: didáctica, tecnología y comunicación, no sólo desde la perspectiva que hay que enseñar a los estudiantes, sino que hay que enseñar a los docentes, porque nos pasa lo mismo que a todos, un grupo de médicos muy prestantes pero que requieren herramientas para poder enseñar.' (UTP)

La preocupación es mayor con los docentes clínicos, donde específicamente es importante hacer la sensibilización y la capacitación porque probablemente no favorezcan mucho el aprendizaje y sí el desaprendizaje de conocimientos y de actitudes aprendidas durante los primeros semestres, relacionadas con el currículo oculto, que impacta la formación de los profesionales [28]. Esto puede explicarse, entre otras razones, por la falta de formación en comunicación, la falta de reflexión sobre el tema durante las prácticas clínicas, que no se hace cuando se discute sobre un diagnóstico o un tratamiento. Además, sólo así se puede conseguir la apropiación de escenarios y estrategias probadas de utilidad en el aprendizaje de la comunicación clínica, como la entrevista simulada, los ejercicios de rol-playing, entre otros [29-31].

'Les estamos dejando a los especialistas a esos estudiantes que sueñan con ser cirujanos plásticos, anestesiólogos, ginecobstetras, etc., que entran en un esquema donde los obligan a desaprender; entonces, solamente vuelven a intentar reflexionar alrededor de la comunicación cuando egresan, pero mientras están en contacto con los especialistas, están perdiendo todo lo que lograron aprender de los otros. (UdeA).

En conclusión:

- El desarrollo de la competencia comunicativa en los profesionales de la salud está presente en las universidades colombianas. Debe hacerse más explícita en los semestres clínicos, con prácticas reflexivas integradas en las discusiones clínicas.

- La capacitación de los docentes clínicos en comunicación se consideró una prioridad para las instituciones participantes.

- Para facilitar el proceso formativo integral es necesario propiciar el acercamiento de los docentes al pensar, al sentir, al ser de los estudiantes, con sus tecnologías, sus potencialidades y también sus dificultades o debilidades.

- Se reconocen carencias en los procesos de evaluación de la competencia en comunicación.

Para fortalecer la formación de la competencia comunicativa en los estudiantes se propone promover el trabajo interdisciplinario e interinstitucional, con eventos académicos, talleres de capacitación, nuevos encuentros sobre avances y proyectos de investigación conjuntos. 
Otra propuesta se dirige a la evaluación de los logros de los estudiantes de acuerdo con los propósitos de formación de cada institución, con actividades como entrevistas con pacientes reales o estandarizados, instrumentos validados y la inclusión de la evaluación de la comunicación en evaluaciones clínicas estructuradas.

La comunicación en su objeto de estudio se interviene desde la lógica de sentidos y significados. En esta orientación valdría la pena reconocerla como mediación pedagógica, como relación que favorece procesos educativos propiciadores de cambios culturales requeridos en la promoción de la salud y la prevención de la enfermedad, es decir, incorporar la comunicación como una competencia que trasciende el manejo de medios y estrategias educativas hacia dinámicas pertinentes al lenguaje y la cultura.

\section{Bibliografía}

1. Bürgi H, Rindlisbacher B, Bader C, Bloch R, Bosman F, Gasser C, et al. Swiss catalogue of learning objectives for undergraduate medical training. 2 ed. Bern: Joint Commission of the Swiss Medical Schools (SMIFK/CIMS); 2008.

2. Simpson JG, Furnace J, Crosby J, Cumming AD, Evans PA, Friedman Ben David M, et al. The Scottish doctor-learning outcomes for the medical undergraduate in Scotland: a foundation for competent and reflective practitioners. Med Teach 2002; 24: 136-43.

3. Shield RR, Tong I, Tomas M, Besdine RW. Teaching communication and compassionate care skills: an innovative curriculum for pre-clerkship medical students. Med Teach 2011; 33: e408-16.

4. Kiessling C, Dieterich A, Fabry G, Holzer H, Langewitz W, Muhlinghaus I, et al. Communication and social competencies in medical education in German-speaking countries: the Basel consensus statement. Results of a Delphi survey. Patient Educ Couns 2010; 81: 259-66.

5. Simpson M, Buckman R, Stewart M, Maguire P, Lipkin M, Novack D, et al. Doctor-patient communication: the Toronto consensus statement. BMJ 1991; 303: 1385-7.

6. Von Fragstein M, Silverman J, Cushing A, Quilligan S, Salisbury H, Wiskin C. UK consensus statement on the content of communication curricula in undergraduate medical education. Med Educ 2008; 42: 1100-7.

7. Borrell-Carrió F, Suchman AL, Epstein RM. The biopsychosocial model 25 years later: principles, practice, and scientific inquiry. Ann Fam Med 2004; 2: 576-82.

8. Tizón JL. El modelo biopsicosocial: la práctica de la teoría es imposible sin una política para la práctica [respuesta del autor] Aten Primaria 2007; 39: 451-3.

9. Clèries X. La esencia de la comunicación en educación médica. Educ Med 2010; 13: 25-31.

10. Moore P, Gómez G, Kurtz S, Vargas A. La comunicación médico-paciente: ¿cuáles son las habilidades efectivas? Rev Med Chile 2010; 138: 1047-54.
11. Salazar OF, Casasbuenas L, Idárraga C. Percepción de la habilidad comunicativa en la entrevista clínica de los estudiantes de medicina de último año. Granada, España; 2012.

12. Palés AJ. Planificar un currículo o un programa formativo. Educ Med 2006; 9: 59-65.

13. Clèries X, De Nadal J, Branda L. Formar en comunicación a los estudiantes de medicina: a propósito de un seminario para profesores universitarios. Educ Med 2006; 9: 2-5.

14. Cáceres MD. Introducción a la comunicación interpersonal. Madrid: Síntesis; 2003.

15. Nolla-Domenjó M. La evaluación en educación médica. Principios básicos. Educ Med 2009; 12: 223-9.

16. Schirmer J, Mauksch L, Lang F, Marvel M, Zoppi K, Epstein R, et al. Assessing communication competence: a review of current tools. Fam Med 2005; 37: 184-92.

17. Boulet J, Rebbecchi T, Denton E, McKinley D, Whelan G. Assessing the written communication skills of medical school graduates. Adv Health Sci Educ Theory Pract 2004; 9: 47-60.

18. Lang F, McCord L, Harvill L, Anderson D. Communication assessment using the common ground instrument: psychometric properties. Fam Med 2004; 36: 189-98.

19. Rider EA, Hinrichs MM, Lown BA. A model for communication skills assessment across de undergraduate curriculum. Med Teach 2006; 28: 27-34.

20. Duffy FD, Gordon GH, Whelan G, Cole-Kelly K, Frankel R, Buffone N, et al. Assessing competence in communication and interpersonal skills: the Kalamazoo II report. Acad Med 2004; 79: 495-507.

21. Blázquez MD, De la Torre I. Redes sociales sobre salud: medicina 2.0. Revistaesalud.com 2012; 8: 1-8.

22. Juanes JA, Velasco MA, Cabrero FJ, Sánchez JM, Rodríguez MJ. Recursos tecnológicos audiovisuales de formación en red: sistemas streaming media y teleinmersivos. TESI 2010; 11 : 214-31.

23. Pauker JL, Richards BF. How medical students and residents describe the roles and characteristics of their influential clinical teachers. Acad Med 2000; 75: 843-5.

24. Salazar OF, Galindo L, Patiño D. El caso de una buena práctica de educación médica. IATREIA 2012.

25. Cabalín D, Navarro N, Zamora J, San Martín S. Concepción de estudiantes y docentes del buen profesor universitario: Facultad de Medicina de la Universidad de La Frontera. Int J Morphol 2010; 28: 283-90.

26. Sogi C, Zavala S, Oliveros MA, Salcedo C. Percepción de formación en entrevista, relación y comunicación médicopaciente. Encuesta en médicos graduados. An Fac Med Lima 2007; 68: 159-67.

27. Triviño X, Sirhan M, Moore P, Reyes C. Formación en educación de los docentes clínicos de medicina. Rev Med Chile 2009; 137: 1516-22.

28. Rodríguez F. Proceso de Bolonia (V): el currículo oculto. Educ Med 2012; 15: 13-22.

29. Baños E, Pérez J. Cómo fomentar las competencias transversales en los estudios de ciencias de la salud: una propuesta de actividades. Educ Med 2005; 8: 216-25.

30. Baribeau D, Mukovozov I, Sabljic T, Eva KV, Delottinville CB. Using an objective structured video exam to identify differential understanding of aspects of communication skills. Med Teach 2012; 34: 242-50.

31. Moral R. Programas de formación en comunicación clínica: una revisión de su eficacia en el contexto de la enseñanza médica. Educ Med 2003; 6: 159-67. 